\section{Zur Lehre von der Spontanheilung der Karzinome.}

Von Hofrat Dr. A. Theilhaber in München.

Das Karzinom galt ebenso wie die Tuberkulose lange Zeit fïr eine unhcilbare Krankheit. Das Dogma von der Unheilbarkeit der Tuberkulose ist schon lange gefallen, an der Möglichkeit der Spontanheilung des Karzinoms wird hellte kaum von irgend jemand nochr gezweifelt. Jode Krankheit ist wohl eintr spontanen Heilung fälig, solange sie nicht $\% 11$ weit vorgeschritten ist. Wie viele Tausende von tuberkulösen Prozessen jedes Jahr 7.11r Ausheilıng kommen, olıne daß deren Träger mit ein€:ın Arıte nälıere Bekanntschaft gemacht haben, das entzieht sich unserer Kenntnis. Etwas Aehnliches, wenn auch im verringerten Maße, gilt vielleicht vom Karzinom. Wenn der Krebs Synıptome macht und diagnostiziert wird, besteht or nicist schon lange. Wir wissen nicht, wie häıfig Krebse in dell ersten Wochen nach ihrer Entstehıng sich wieder zurückbilden. Wird das Karzinom diagnostiziert. dann ist es gewöhnlich sehon älter, nicht neehr so leicht einer spontanen Riickbildung fähig und wird gewölınlich operiert. Wird es ausnahmsweise nicht operiert und bildet es sich in seltenen Fällen doch noch einmal zurïck, so glaubt der Ar\%t, cine falsche Diagnose gestellt zu haben. Er publiziert den Fall gewöhnlich nicht. Trotz aller dieser erschwercnden Unstände unifaßt die Kasuistik der in den letz.ten Jahrzehnten beobachteten Spontanheilungen von vorgeschrittenen Karzinomen jetzt schon etwa 200 Fälle. Das eingehende Studium dieser Beobachtungen ist sehr wïnsehenswert. Anf diesem Wege werden wir vielleicht den Modus der Naturhcilıng z.um Teil kennen lernen und möglicherweise auf einen Weg hingewiesen werden, dessen Beschrciten das Zustandekonmen der Naturheilung erleichtert. Von diesem Gesiclitspunkt aus halte ich es nicht fiir unniitz, wenn ich kurz iiber einige derartige Beobachtungen referiere.

Bei Totalexstirpationen des Uterus wegen vorgeschrittener Karzinome ist es mir, wic wohl den meisten Operateuren, zu wiederholten Malen passiert, $\mathrm{da} B$ sich kleine karzinomatöse Infiltrate in weiter Entfernung vom Uterus im Bindegewebe etc. zerstrent vorfanden, soda $\beta$ deren vollständige Entfernung unmöglich war. In der Regel war schon nach sehr kurzer Zeit das Rezidiv da. In drei solehen Fällen trat dagegen völlige Heilung cin, und ich habe in der letzten Zeit Ge. legenheit gehabt, diese drei Fälle noch zu untersuchen und nich vou ihrem Wohlbefinden und von der anatomischen Gesundheit der Beckenorgane zu üherzeugen. Der älteste dieser Fälle wurde im Dezenıber 1905 , der letzte in Mai 1908 operiert.

Den crsteu Fall, in dem ich nicht cinnıal den Cterns exstirpierte und in dem doch die Neubildung sich vollkommen zurückbildete, beob. achtete ieh im Anfange dieses Jahres: Frau H., 56 Jahre alt, scit vier Jahren in der Menopausc, hat seit einem Jahre wieder unregelmäBige Blı. tungen. Bei der Lntersuchung findet sich äußerer Mutternuund geöffnet - in der Cervix z.ahlreiche geschwiirig zerfallene Protuberanzen fiihlbar. Uterus verdickt, $10 \mathrm{~cm}$ lang. Links vom Uterus, im Parametriım, eiı pflaumengroßer, harter, mit dem Uterus und den Beckenknochen verwachsencr Tumor. Rechts vom Uterus eine kleincre Infiltration in Parametrium. Hinter dem Cterus im Donglas diffuse Infiltrationen. Der Uterus ist absolut unbeweglich. Ans dem Muttermund flicBt eine scheußlich stiukende Jauche in großer Menge. Patientin ist sehr heruntergekoumen, hoclygradig anämisch. Mikroskopische Untersuchung eines aus. gescliabten Partikels ergibt Karzinom. Es wurde cine Ausschabung und Thermokanterisation vorgenommen. Nach acht Tagen wurde eine Nach. kur begonnen, dic in folgenden bestand: Durch intravaginale Heißluft. behandlıng ınittels Fön, durch heiße Einspülıngen und heiße Sitzbäder wurde der Uterus hyperämisiert. Außerdem wurde sechs Wochen lin dırch täglich, später jedın zweiten Tag subkutan Natr. cacodylic. $(0,05)$ eingesprit\%t; da\%wischen wurden fuinfmal Iujektionen von je 4 r.n nach uneinen Angaben hergestellten Uterusextraktes genıacht

Der AusfluB verringcrte sich, das Aussehen der Patientin wurdc besser, der Kräftezustand hob sicl. Dic Infiltrate im Parametrium bildeten sich zurück, nach fünf Monaten war der AusfluB vollständig verschwunden, die Cervix vollständig veruarbt, der Muttcrmund wieder verengt, del Uterus war von normaler Größe. die Infiltration in der Umgebung des Cterus völlig bescitigt, das Körpergewicht hatte wesent. lich zugenoummen. in jedem Monate 11 m 3- 4 Pfund, die Patientin fiihltc sieh vollständig wolll und befiudet sieh jetzt in den Alpen.

Recht interessant ist anch wohl folgcuder Fall: Fran M. konsul. ticrte mich im Mai 1910 wegen vorgeschrittenen Karzinoms der Manıma, das bereits mit de.ln Pectoralis verwachsen war, die Achscldrüsen waren stark infiltriert, es bestand eine alte Verdichtung der rechten Lungenspitze. Ich cntfernte dir Manma, den Pectoralis, die Arhseldrüsen ctc. Nach vier Wochen kanı sie wegen Schmerzen im Arme; der Vorderarm war hochgradig ödematös; in der Achseldriise fiihlte man eine pflaumen. große Drïsensehwellung; es wurde Biersche Staunng der Narbe und der Achscl vorgenonınıen und täglich wicderholt. Nach einigen Wochen waren Schmerzen, Dedem und Driisensehwelluug verscliwunden. $\mathrm{Pa}$. ticntiu kam nicht ıneh!: - Mitte Dezember kaul die Kranke wieder. Der Achseldriisentumor war vou neuem aufgetreten, der Arm war wieder hochgradig geschwollen. Es wurde nun die Behandlung mittels Heiß luft anfgenonımen. nacl 1 vier Wochen war das Oedem vollständig ge. schwundell, nach zwei Monaten war auch nicht dic Spur einer Lymph. driisenschwollung mehr z.lu fiihlen. Die Behandlung wurde fortgesetzt, Patientin befand sich andauernd wohl, 13/4 Jahre nach der Operation bekanı sie cin klein'ss Pleuraexsudat, das sich jedoch bald zurückbildete und das wahrseheinlich Folge einer alten tuberkulösen Spitzenaffektion war. Die Brustgegend und die Achselhöhle blieben frei von jeglicher Anschwellung.

In einem Sitzungsbericht der Gesellschaft der Acrzte in Wien vom 23. Juni 1911 ist auch eine kurze Mittcilung iiber cinen Vortrag Hochencggs enthalten, in dem dicser ebenfalls für energische Nach. kuren nach Karæinomoperationen eintritt. Er hat daselbst über gute Erfolge mit Nachkuren unittels Aenderung der Lebensweise und Sonnenlichtbchandlung berichtet. Auch Brosch ${ }^{1}$ ) berichtet iiber einen $\mathrm{Pa}$ tienten, bei dem von inehreren renommierten Aerzten die Diagnose eines hochsitzenden Krebses der Flexura sigmoidea gestellt war. Da der Patient sich zu einer Operation nicht entschließen konnte, behandelte ihn Brosch nittels subaqualer, 33 ${ }^{\circ} \mathrm{R}$ heißer Darmspülungen ${ }^{2}$ ). Außerdem wurden, $11 \mathrm{~m}$ eine alkalische Reaktion des Darminhalts herbeizuführen, Bleibeklismata mit anfangs 5 - und später $10 \%$ iger $\mathrm{Na}$. triumbikarbonatlösung angewandt.. Nach dreimonatiger Bchandlung war der Tumol auf ungefähr ein Drittel seiner ursprünglichen Größe gesunken.

Der Patieut sicht jetzt (nach vicr Monaten) gesund als, lat scine Leistungsfähigkeit wieder erlangt. Er fühlt sich derart wohl, daß er sich für vollkommen gesund halten würde, wenn nielit der klẹine Tunıorrest noch immer palpabel wäre. Dic Blıtungen sind dauernd ausgeblieben. Das Körpergewicht hat $11 \mathrm{~m} 3 \mathrm{~kg}$ zugenommen.

Nach meinen Untersuchungen entwickelt sich das Karzinom nicht in normalen Geweben. Die dem Krebs vorausgehenden Störungen sind meist charakterisiert durch Atrophie der subepithelialen Gewcbe. Bei der Untersuchung in der Umgebung beginnender Karzinome fand ich Verringerung der $\mathrm{Zahl}$ und der Größe der Zellen, außerdem Anämie (spärliche, häufig stenosierte und obliterierte Gefäße). Einer der Gründe für nıanche Rezidive nach Operationen ist nıeines Erachtens der Umstand, daß die Operationsnarbe auch ein in schlechtem Ernährungszustand sich befindendes Gewebe ist. Hieraus zog ich den SchluB, daB die Rezidive nach Operationen vielleicht vermindert werden könnten, wenn es gelingen würde, die Ernährung der Narbe zu heben. Faute de mieux habe ich $^{3}$ ) zunäclist folgende Mittel in Anwendung gebracht: Hebung des Allgemeinzustandes durch Gebirgsluft, Seebäder und andere klimatische Kuren, Stauung und Hyperämisierıng der Narbe durch Heißluftbehandlıng, Fibrolysin, nach Cterusexstirpation Oophorin. Rationell scheint mir auch noch Hocheneggs Vorschlag, Sonnenbäder anzluwenden, zum Teil, um die Ernähl'ıng der Narbe zı hcben, zum Teil, um den Kräftez.ustand im allgemeinen z.u bessern.

Dic unvollständigen Karzinomoperationen, bei denen icl cine radikale Heilung erzieltc, hatten das Gemcinsame, daß bei ihucn der Blutverlust während der Operation, da die Instrumente in dom karzinomatösen Gewebe nicht hielten, groß war.

Ich glaubc, daB die Möglichkeit eincs Kausalzusammenhanges zwischen starkem Blutverlust und Radikalheilung nicht völlig auszuschlicß3en ist. Nach einer ausgiebigen Blutentziehung wird die Blut-

1) Medizinische Klinik 1912, Yo. 17. - 2) Brosch und Auf. schnaiter, Das subaquale Innenbad, Leipzig, Dcuticke, 1912. -$\left.{ }^{3}\right)$ Sitzung des Aerz.tlichen Vereins München, Dezember 1909. 
zirkulation im Körper lebhafter, der Lymphstrom ninımt 7.u, die Schweißsekretion wird vermehrt, es komnit zur Hyperleukozytose, auch die Zahl der roten Blutkörperchen ist nach meinen Untersuchungen nach einigen Wochen meist größer als vor dem Blutverlust. Es ist deshalb schou denkbar, daß ein beträchtlicher Blutverlust ein Anregungsmittel für die Resorption zurïckgebliebener Karzinom\%ellen ist. Ich habe deshalb im letzten Jahre der obenerwähnten Behandlung in der zweiten Woche nach der Operation noch cinen Ade:la B von $400--500 \mathrm{cem}$ hinzugefügt.

In den $2 \frac{1}{2}$ Jahren, in denen ich diese Nachbehandlung zur Anwendung brachte, haben sich meine Resultate in bezug auf Rezidive beträchtlich gebessert. Allerdings sind meine Ziffern noch nicht so groß, daß der Schluß, ,post hoc ergo propter hoc" absolut einwandfrei gemacht werden könnte. Doch kann ich gegenüber den Einwendungen einzelner Aerzte bestimmt sagen, da B ein schädlicher Einfluß dieser Nachbchandlung gan\% bestimmt nicht stattfand.

Die Hypcrämisicrung dürfte vielleicht auch bei einzclnen Fällen von inoperablem Karzinom die Naturheilung untcrstützen. Die Grïnde für die Arsenikbehaudlung werde ich später publizieren. Dic Erfolge in dem initgeteilten Falle ermutigen jedenfalls da\%u, die Kombination von Hyperämisierung und Cacodvleinspritzungen weiter zu versuchen. Es läßt sich nicht entscheiden, ob und wievicl dic Einspritzungen unseres Extraktes \%u dem Erfolge mit beigetragen haben. Bei einer Reihe andere sehr vorgeschrittener Fälle von Karzinom, wo die Kombination mit Cacodyl und Hyperänisicrung unterblieb, traten nur voriibergehende Besserungen allf.

Zum Schlusse ziticre ich einige Sätze aus der vorhin erwähnten Mitteilung von Broseh. Er sagt:

,Theilhaber wendet als lokale Maßnahmen zur Vernichtung zurückgebliebener Krebskeime Fibrolysin, Hyperänisierung der (Ope. rations.) Narben durch Stauung, Heißluft und dergleichen an und hofft auf Frsinnung neucr technischer Maßnahmen in dieser Richtung und weitere Besserung in der Krebsbehandlung, falls es gelingen sollte, mehr Aerzte für diesen Weg zu interessicren. Wenn uuscre Zeilen dazu beitragen, das Interesse der Acrzte an der Gangbarmachung dieses neuen, durch die Namen Stahr, Hochenegg, 'Theilhaber markierten Krebsbehandlungsweges 7.4 erhöhen, dann haben sie ihren eigentlichen Zweck, neuc Mitarbeiter zu werben, reichlich erfüllt, denn mit der wachsenden Zahl der Sucher steigen auch die Chancen für wertvolle Funde.“" 